\title{
Klotho alleviates chronic intermittent hypoxia-induced genioglossus myocyte apoptosis by inhibiting endoplasmic reticulum stress
}

\author{
ZHEN XUE ${ }^{1}$, WENXIAO DING ${ }^{2}$, LUYAO GE ${ }^{2}$ and QIANG ZHANG ${ }^{2}$ \\ ${ }^{1}$ Department of ENT \& HN Surgery, Lishui District People's Hospital of Nanjing, Nanjing, Jiangsu 211200; \\ ${ }^{2}$ Department of Respiratory Medicine, Zhongda Hospital Affiliated to Southeast University, \\ Nanjing, Jiangsu 210009, P.R. China
}

Received September 3, 2020; Accepted February 17, 2021

DOI: $10.3892 /$ etm.2021.10140

\begin{abstract}
Chronic intermittent hypoxia $(\mathrm{CIH})$ has been shown to induce cell apoptosis in multiple organs of the human body. The present study aimed to assess the effects of exogenous klotho on CIH-induced genioglossus muscle injury, as well as the involvement of endoplasmic reticulum stress (ERS) in this process. A total of 36 adult C57BL/6 male mice were assigned to normoxia control (NC), $\mathrm{CIH}$ and $\mathrm{CIH}+$ klotho groups ( $\mathrm{n}=12$ mice/group). ELISA was performed to detect the level of klotho protein in the serum and in the genioglossus muscle tissue samples. Apoptosis was evaluated using the TUNEL assay. Reactive oxygen species (ROS) levels were quantified using a dihydroethidium assay kit, and the protein and mRNA levels of ERS-associated proteins (namely, glucoseregulated protein $78, \mathrm{C} / \mathrm{EBP}$ homologous protein, cleaved caspase-12 and cleaved caspase-3) in genioglossus samples were assessed using immunoblot assay and reverse transcription-quantitative PCR, respectively. Compared with the NC group, the quantities of klotho protein in the serum and genioglossus muscle tissue samples in the CIH group were significantly decreased, whereas the apoptotic rate, ROS levels and protein and mRNA levels of the ERS-associated proteins in the genioglossus
\end{abstract}

Correspondence to: Dr Qiang Zhang, Department of Respiratory Medicine, Zhongda Hospital Affiliated to Southeast University, 87 Dingjiaqiao Road, Nanjing, Jiangsu 210009, P.R. China E-mail: 13952036589@163.com

Abbreviations: $\mathrm{CIH}$, chronic intermittent hypoxia; ERS, endoplasmic reticulum stress; NC, normoxia control; ROS, reactive oxygen species; RT-qPCR, reverse transcription-quantitative PCR; OSAHS, obstructive sleep apnea-hypopnea syndrome; IRE1, including inositol-requiring enzyme; PERK, PKR-like endoplasmic reticulum kinase; GRP78, glucose-regulated protein 78; IGF-1, insulin-like growth factor-1; ASK-1, apoptotic signal-regulated kinase-1

Key words: genioglossus muscle, klotho protein, chronic intermittent hypoxia, apoptosis, ROS, endoplasmic reticulum stress muscle were significantly increased. Following supplementation with exogenous klotho protein, the klotho protein levels in the serum and genioglossus muscle tissue of mice were found to be markedly increased, and the apoptotic rate, ROS levels and protein and mRNA levels of the ERS-associated proteins in the genioglossus muscle were decreased compared with those in the $\mathrm{CIH}$ group. Taken together, the results of the present study have demonstrated that exogenous klotho may inhibit apoptosis of genioglossus myocytes in mice by inhibiting ROS-associated ERS.

\section{Introduction}

Obstructive sleep apneahypopnea syndrome (OSAHS) poses a serious threat to human health, affecting 3.7-97.3\% of Asian adults (1). The pathophysiological mechanism underpinning OSAHS includes repeated upper airway stenosis and collapse in the sleep state, resulting in recurrent apnea, hypopnea and chronic intermittent hypoxia (CIH) during sleep (2). Based on current knowledge, OSAHS is considered to be closely associated with structural stenosis of the airway, reduced muscle tension and abnormal function of the upper airway. The genioglossus muscle, as the main upper airway dilator, is crucial for ensuring that the upper airway remains unobstructed, and is referred to as the 'safety muscle of the upper airway' (3). Zhang et al (3) reported that $\mathrm{CIH}$ induced genioglossus myocyte apoptosis through activating endoplasmic reticulum (ER) stress (ERS). Wang et al (4) reported that CIH induced ROS production and cell apoptosis in the genioglossus through downregulating and upregulating mitophagy by adiponectin to improve the $\mathrm{CIH}$-induced genioglossus myocyte injury. It was previously reported that stimulation of rats with $\mathrm{CIH}$ led to an increase in the fatigability of upper airway muscles and caused hypoadiponectinemia, disrupted genioglossal ultrastructure and mitochondrial dysfunction $(5,6)$.

Mitochondrial dysfunction, in turn, affects the major pathways implicated in the pathophysiology of airway disease, including airway contractility, response to oxidative stress and apoptosis (7). In total, three apoptotic pathways have been described: The ER, mitochondrial and death receptor pathways (8). External stressors can cause unfolded or misfolded 
proteins to accumulate in the ER, resulting in ERS. In order to maintain homeostasis, the unfolded protein response (UPR) pathway activates three transcription factors, including inositolrequiring enzyme (IRE1), type I PKRlike endoplasmic reticulum kinase (PERK) and activating transcription factor 6 (ATF6) $(9,10)$. Under normal conditions, these three transcription factors are bound to glucose-regulated protein 78 (GRP78) and remain inactive. Under stress conditions, however, GRP78 and the transcription factors dissociate, leading to activation of the UPR (11). The activated IRE1, PERK and ATF6 pathways all upregulate $\mathrm{C} / \mathrm{EBP}$ homologous protein $(\mathrm{CHOP})$, which, in turn, regulates $\mathrm{Bcl}-2$ family proteins, increasing the rate of synthesis of the pro-apoptotic protein Bim, reducing the synthesis of the anti-apoptotic protein $\mathrm{Bcl}-2$ and causing mitochondrialassociated apoptosis $(12,13)$. In addition, during ERS, caspase-12 is activated on the ER membrane and, as an ERS apoptosis-specific protein, activates downstream caspase-3, leading to apoptosis $(14,15)$. A previous study by our research group demonstrated that $\mathrm{CIH}$ upregulates ERS-associated proteins in rat genioglossus myocytes, activates ERS-associated apoptosis pathways and causes genioglossus dysfunction (3).

The klotho gene is located on chromosome $13 \mathrm{q} 12$, and encodes two types of proteins: Membrane-bound and secreted klotho proteins. A previous study revealed that secreted klotho protein exerts cytoprotective effects, inhibiting oxidative stress and apoptosis (16). In exploring the underlying mechanisms, Maekawa et al (17) determined that klotho both promoted MEK/ERK pathway activation and led to a significant decrease in apoptosis of human umbilical vein endothelial cells stimulated by hydrogen peroxide. Furthermore, Yamamoto et al (18) demonstrated that in vitro supplementation of soluble klotho led to a marked reduction in paraquat-induced lipid peroxidation in HeLa cells via inhibiting the insulin-like growth factor-1 (IGF-1) pathway. A previous study also revealed reduced klotho protein levels in serum samples collected from patients with obstructive sleep apnea syndrome (OSAS), and the klotho protein level was found to be negatively correlated with disease severity (19). Navarro-González et al (20) demonstrated that the protective role of pentoxifylline was associated with increased levels of klotho in patients with diabetes and chronic kidney disease. Therefore, taken together, the finding of these studies suggested that supplementary klotho may protect against OSAS-induced injury; however, the mechanisms underpinning these effects have yet to be elucidated. To meet this end, the present study aimed to assess the effects of exogenous klotho on $\mathrm{CIH}$-induced genioglossus muscle injury in a mouse model, and determine the involvement of ERS in this process.

\section{Materials and methods}

Mice and grouping. In total, 36 male adult C57BL/6 mice, aged 8 weeks, weighing 18-20 g, were purchased from and housed at the Animal Center of Southeast University. The animals were provided with access to water and standard food ad libitum under a 12 -h light/dark cycle at $24^{\circ} \mathrm{C}$ and $60 \%$ humidity. The present study was approved (approval no. 201704025) by the Experimental Animal Ethics Committee of Southeast University. The mice were assigned to three groups according to the random number table method as follows: The normal control (NC), $\mathrm{CIH}$ and $\mathrm{CIH}+$ klotho groups $(\mathrm{n}=12$ mice per group; the different treatments and conditions of the experimental groups are explained in detail below).

Establishment of the mouse CIH model and intraperitoneal injection of klotho. The mouse hypoxia box (Nanjing Xinfei Analytical Instrument Co., Ltd.) was used to establish the mouse CIH model, as described previously (6). Briefly, the hypoxia cycle time was set to $1 \mathrm{~min}$, and the chamber was filled with nitrogen for the first $30 \mathrm{sec}$ to reduce oxygen concentration to $6-7 \%$; subsequently, air was allowed into the chamber for the second $30 \mathrm{sec}$ to gradually increase the oxygen concentration to $21 \%$. A total of 60 cycles were performed per h, thereby simulating severe human OSAHS. The $\mathrm{CIH}$ and $\mathrm{CIH}+$ klotho groups underwent intermittent hypoxia treatment for 8 h (8:00 a.m. - 4:00 p.m.) every day, for a total of 12 weeks, whereas the $\mathrm{NC}$ group was placed in the hypoxia box that was only filled with air in parallel.

Recombinant mouse klotho protein was purchased from R\&D Systems, Inc. and dissolved in sterile normal saline solution $(1 \mu \mathrm{g} / \mathrm{ml})$. Mice in the $\mathrm{CIH}+\mathrm{klotho}$ group received an intraperitoneal injection of klotho protein (10 $\mu \mathrm{g} / \mathrm{kg} /$ day) $(21,22)$, whereas the $\mathrm{NC}$ and $\mathrm{CIH}$ groups were administered normal saline $(0.5 \mathrm{ml} /$ day $)$ intraperitoneally during model establishment.

After modeling, the mice were anesthetized with $1 \%$ pentobarbital $(50 \mathrm{mg} / \mathrm{kg})$ via intraperitoneal injection, and $1 \mathrm{ml}$ blood was collected by cardiac puncture and centrifuged at $1,500 \mathrm{x} \mathrm{g}$ for $15 \mathrm{~min}$ at $4^{\circ} \mathrm{C}$ for serum preparation. The resulting serum was stored at $-80^{\circ} \mathrm{C}$ prior to analysis. The mice were euthanized by exsanguination after anesthesia with pentobarbital $(1 \% ; 50 \mathrm{mg} / \mathrm{kg})$, the genioglossus muscle was isolated, and a portion of the muscle was placed in $4 \%$ paraformaldehyde. After fixation for $48 \mathrm{~h}$ at $4{ }^{\circ} \mathrm{C}$, the samples were dehydrated with xylene, waxed, embedded in paraffin and cut into $5-\mu \mathrm{m}$ sections for subsequent analysis. The remaining genioglossus muscle samples were stored at $-80^{\circ} \mathrm{C}$.

Detection of klotho protein levels in serum and genioglossus muscle samples. ELISA was performed to detect the serum klotho protein levels in the mice using the klotho protein kit (cat. no. DL-KL-Mu; Wuxi Donglin Sci \& Tech Development Co., Ltd.), following the manufacturer's protcols. For assessment of the klotho protein level in the tissue, $20 \mathrm{mg}$ fresh genioglossus tissue was placed in $500 \mu \mathrm{l}$ PBS, homogenized, and cleared by centrifugation at 5,000 x $\mathrm{g}$ for $5 \mathrm{~min}$. The supernatant was collected and tested as described for the serum samples. Klotho protein levels in serum and tissue samples were assessed by reading the absorbance at $450 \mathrm{~nm}$ on a spectrophotometer.

Apoptosis detection. A TUNEL assay was performed to detect apoptosis of genioglossus myocytes in mice. The TUNEL kit (cat. no. 11684817910) was purchased from Roche Diagnostics $\mathrm{GmbH}$ and experiments were performed according to the manufacturer's instructions. The genioglossus sections were treated with protease $\mathrm{K}(20 \mu \mathrm{g} / \mathrm{ml})$ for $15 \mathrm{~min}$ at room temperature after deparaffinization and rehydration. The sections were then treated with the TUNEL reaction 
Table I. PCR primers for detecting endoplasmic reticulum stress-related genes in genioglossus muscle samples.

\begin{tabular}{ll}
\hline Gene & \multicolumn{1}{c}{ Primer sequences $\left(5^{\prime}-3^{\prime}\right)$} \\
\hline GRP78 & Forward: CTCGGATCCACCATGATGAAGTTCACTGTGGTG \\
CHOP & Reverse: TGCTCTAGAGCTCAACTCATCTTTTTCTGATG \\
& Forward: CTCGCTCTCCAGATTCCAGT \\
GAPDH & Reverse: CTGCTCCTTCTCCTTCATGC \\
& Forward: AGCAGTCCCGTACACTGGCAAAC \\
& Reverse: TCTGTGGTGATGTAAATGTCCTCT
\end{tabular}

GRP78, glucose-regulated protein 78; CHOP, C/EBP homologous protein.

mixture for $1 \mathrm{~h}$ at $37^{\circ} \mathrm{C}$ in the dark and humidified atmosphere. Next, the sections were treated with DAPI for $5 \mathrm{~min}$ at room temperature in the dark before being mounted in neutral resin. A fluorescence microscope was used for observation of the cells. Blue and green signals represented normal and apoptotic nuclei, respectively. In total, five random fields per tissue section were captured at x200 magnification. The extent of cell apoptosis was quantified using ImageJ 1.52 software (National Institutes of Health).

Hematoxylin and eosin $(H \& E)$ staining. The genioglossus muscle was fixed in $4 \%$ paraformaldehyde for $48 \mathrm{~h}$ at $4^{\circ} \mathrm{C}$, after which the samples were dehydrated, embedded in paraffin and cut into $5-\mu \mathrm{m}$ sections prior to H\&E staining. Subsequently, the sections were deparaffinized, rehydrated and stained. Each section was subjected to hematoxylin and eosin staining (1 min each) at room temperature. Finally, the sections were observed under a light microscope (magnification, x400) after dehydration and preparation of cover slips.

Western blot analysis. Total protein was extracted from the genioglossus muscle using the protein extraction kit (cat. no. KGP2100) of Nanjing KeyGen Biotech Co., Ltd. Genioglossus muscle tissue samples were homogenized on ice in lysis buffer containing $10 \mu \mathrm{l}$ phosphatase inhibitor, $1 \mu \mathrm{l}$ protease inhibitor and $10 \mu \mathrm{l} 100 \mathrm{mM}$ PMSF. The supernatant was then collected by centrifugation at $12,000 \times \mathrm{g}$ and $4^{\circ} \mathrm{C}$ for $5 \mathrm{~min}$. The protein concentration was measured using the BCA method (Thermo Fisher Scientific, Inc.). Equal amounts of protein $(20 \mu \mathrm{g})$ were resolved by SDS-PAGE $(10 \%)$ followed by electro-transfer onto a PVDF membrane (Roche Diagnostics). In total, 5\% bovine serum albumin (Beyotime Institute of Biotechnology) was used to block the membranes for $1 \mathrm{~h}$ at room temperature; subsequently, the samples were successively incubated with primary (overnight at $4^{\circ} \mathrm{C}$ ) and secondary $\left(37^{\circ} \mathrm{C}\right.$ for $1 \mathrm{~h}$ ) antibodies diluted in $5 \%$ bovine serum albumin in TBS with 0.1\% Tween-20 at pH 7.6. Antibodies against CHOP (cat. no. 2895; monoclonal antibody), GRP78 (cat. no. 3183; rabbit polyclonal antibody), GAPDH (cat. no. 5174; rabbit monoclonal antibody) and cleaved caspase-3 (cat. no. 9664; rabbit monoclonal antibody) were purchased from Cell Signaling Technology, Inc., whereas the antibody against cleaved caspase-12 (cat. no. ab62463; rabbit polyclonal antibody) was obtained from Abcam. The horseradish peroxidase-linked secondary antibodies
(Anti-mouse, 1:1,000, cat. no. 7076; Anti-rabbit, 1:1,000, cat. no. 7074) were purchased from Cell Signaling Technology. ECL solution was evenly applied on to the PVDF membrane, and the membranes were subsequently photographed with a fluorescence imaging machine. The densitometric evaluation of the bands was performed using Image Lab ${ }^{\mathrm{TM}} 6.0$ software (Bio-Rad Laboratories, Inc.).

Detection of the mRNA levels of ERS-associated genes in genioglossus muscle samples. Reverse transcriptionquantitative PCR (RT-qPCR) analysis was performed to detect the mRNA expression levels of GRP78 and CHOP. Total RNA was obtained by lysing the genioglossus muscle with TRIzol ${ }^{\circledR}$ lysis buffer (Thermo Fisher Scientific, Inc.). Subsequently, $1 \mu \mathrm{g}$ total RNA was reversetranscribed using the Transcriptor First Strand cDNA Synthesis Kit (cat. no. 04897030001) of Roche Diagnostics $\mathrm{GmbH}$ using the following protocol: $30 \mathrm{~min}$ at $55^{\circ} \mathrm{C}$ and at $85^{\circ} \mathrm{C}$ for $5 \mathrm{~min}$. After mixing the primers of GRP78, CHOP, and GAPDH (for the sequences, see Table I; Invitrogen, Thermo Fisher Scientific, Inc.), $1 \mu \mathrm{g}$ cDNA, and Power SYBR ${ }^{\circledR}$ Green PCR Master Mix (cat. no. 4367659; Applied Biosystems; Thermo Fisher Scientific, Inc.), RT-qPCR was performed on an ABI7900 instrument, and the thermocycling conditions were as follows: Initial denaturation for $10 \mathrm{~min}$ at $95^{\circ} \mathrm{C}, 40$ cycles of $15 \mathrm{sec}$ at $95^{\circ} \mathrm{C}$ and $1 \mathrm{~min}$ at $60^{\circ} \mathrm{C}$. The $2^{-\Delta \Delta \mathrm{Cq}}$ method (23) was used for quantitative analysis of the mRNA levels.

Detection of reactive oxygen species (ROS). OCT (cat. no. 4583; Sakura Finetek USA, Inc.) embedding solution was used to embed fresh genioglossus muscle tissue samples, which were cut using a frozen microtome for subsequent use. Frozen sections $(6 \mu \mathrm{m})$ were treated with dihydroethidium assay kit (cat. no. S0063; Beyotime Institute of Biotechnology) according to the manufacturer's instructions, prior to analysis with fluorescence microscopy (magnification, x200). In total, five random fields per tissue section were captured. ImageJ 1.52 software (National Institutes of Health) was used to detect the fluorescence intensity, which represented the reactive oxygen species level.

Statistical analysis. GraphPad 7.0 software (GraphPad Software, Inc.) was used for data analysis. Data are presented as the mean \pm standard deviation, and were analyzed by oneway ANOVA followed by the Student-Newman-Keuls 

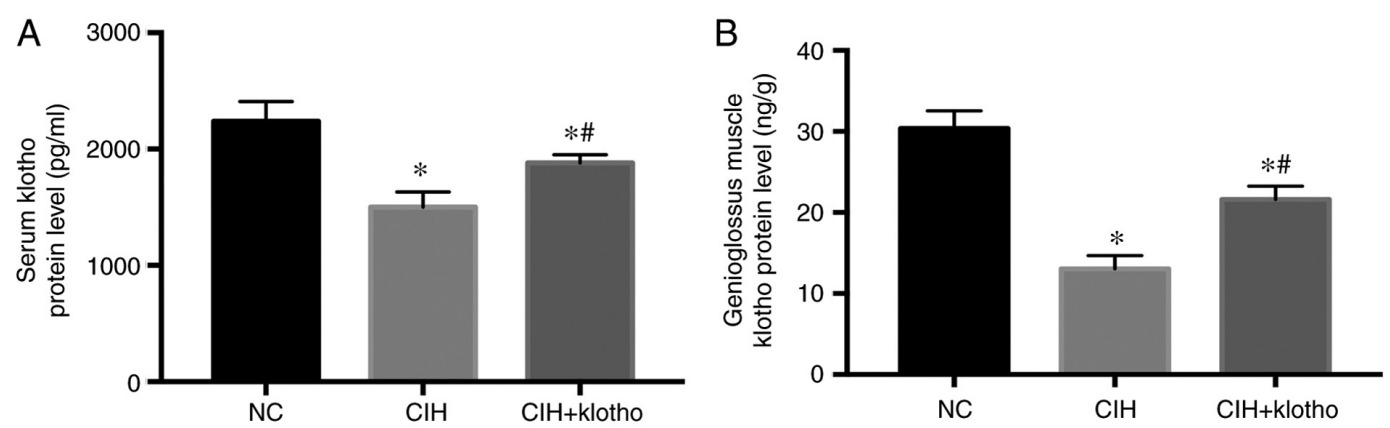

Figure 1. Serum and genioglossus muscle tissue klotho protein levels. Klotho protein levels in the serum and genioglossus muscle tissue samples were assessed by ELISA. (A) Klotho protein level of serum. (B) Klotho protein level of genioglossus muscle. . "P $<0.05$ vs. CIH group; ${ }^{\text {P }}<0.05$ vs. NC group. NC, normoxia control; $\mathrm{CIH}$, chronic intermittent hypoxia.

post hoc test. $\mathrm{P}<0.05$ was considered to indicate a statistically significant difference.

\section{Results}

Serum and genioglossus muscle klotho protein levels. At 12 weeks after initiating establishment of the mouse $\mathrm{CIH}$ model, the levels of the klotho protein in the serum and genioglossus muscle samples of the C57BL/6 mice were found to be significantly decreased in the $\mathrm{CIH}$ group compared with those in the NC group $(\mathrm{P}<0.05)$. Following exogenous klotho protein administration, however, the levels of klotho protein in the serum and genioglossus muscle tissue were significantly increased compared with those in the CIH group $(\mathrm{P}<0.05)$, although they remained lower in comparison with those in the $\mathrm{NC}$ group $(\mathrm{P}<0.05$; Fig. 1$)$. These results suggested that klotho protein injected intraperitoneally was distributed systemically, including its delivery to the genioglossus muscle tissue.

Apoptosis of genioglossus myocytes. After 12 weeks of $\mathrm{CIH}$ modeling, the apoptotic rate of the genioglossus myocytes was found to be significantly higher in the $\mathrm{CIH}$ group $(7.633 \pm 0.1672 \%)$ compared with that in the NC group $(0.813 \pm 0.0719 \% ; \mathrm{P}<0.05)$. Following administration of the klotho protein, however, the apoptotic rate was markedly reduced $(4.609 \pm 0.3164 \%)$ compared with that in the $\mathrm{CIH}$ group $(\mathrm{P}<0.05)$, although this remained higher compared with the control value $(\mathrm{P}<0.05$; Fig. 2$)$. These results suggested that administration of exogenous klotho protein could alleviate apoptosis in genioglossus myocytes.

Histological changes in the genioglossus. After 12 weeks of $\mathrm{CIH}$ modeling, the H\&E staining experiments revealed no significant differences in terms of histological changes among the three groups (Fig. 3).

Gene expression levels of ERS-associated molecules in genioglossus muscle samples. After 12 weeks of $\mathrm{CIH}$ modeling, the GRP78 and CHOP mRNA levels in the genioglossus muscle samples were significantly increased in the CIH group compared with those in the NC group $($ all $\mathrm{P}<0.05)$. Compared with the $\mathrm{CIH}$ group, the $\mathrm{CIH}+$ klotho group revealed markedly reduced mRNA levels of GRP78 and CHOP (all P<0.05). The CHOP mRNA levels

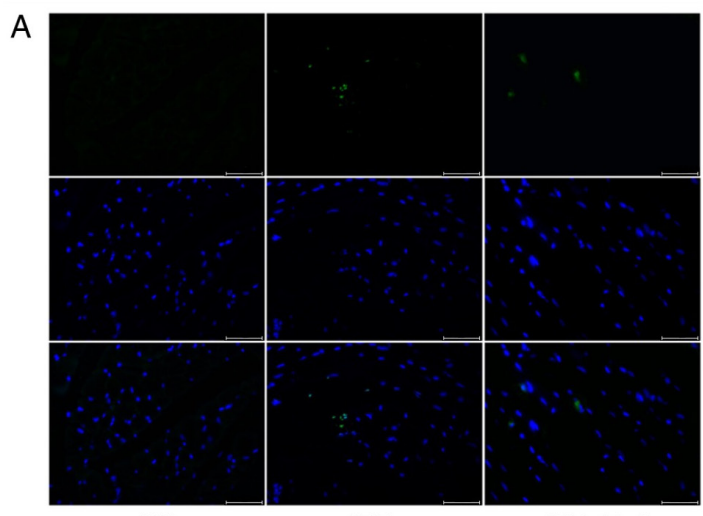

NC $\quad \mathrm{ClH} \quad \mathrm{ClH}+$ klotho

B

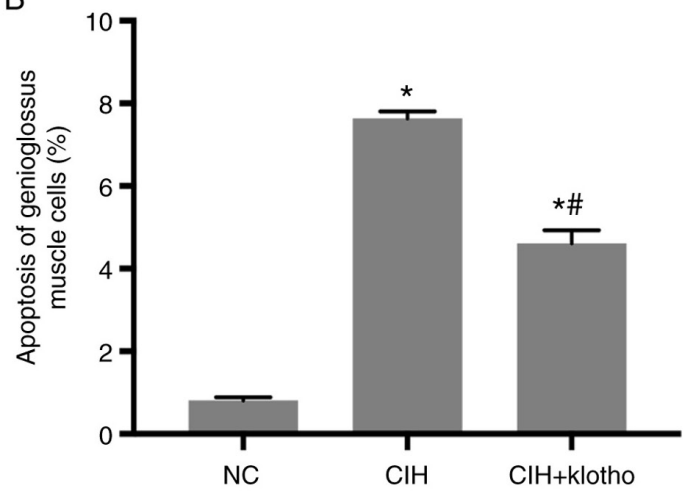

Figure 2. Apoptosis of genioglossus myocytes. Apoptotic cells were quantitated by TUNEL assay. (A) Micrographs showing normal and apoptotic nuclei as blue and green signals, respectively. Scale bar, $50 \mu \mathrm{m}$. (B) Quantitation of A. ${ }^{*} \mathrm{P}<0.05$ vs. $\mathrm{CIH}$ group; ${ }^{\text {}} \mathrm{P}<0.05$ vs. $\mathrm{NC}$ group. NC, normoxia control; $\mathrm{CIH}$, chronic intermittent hypoxia.

in the $\mathrm{CIH}+$ klotho group remained higher compared with those of the NC group $(\mathrm{P}<0.05)$, whereas the GRP78 gene expression levels in the $\mathrm{CIH}+$ klotho and $\mathrm{NC}$ groups were comparable (P>0.05; Fig. 4).

Protein expression levels of ERS-associated molecules in genioglossus muscle samples. After 12 weeks of $\mathrm{CIH}$ modeling, the protein expression levels of ERS-associated proteins (GRP78, CHOP, cleaved caspase-12 and cleaved caspase-3) in the genioglossus muscle samples were significantly higher in the $\mathrm{CIH}$ group compared with those in the $\mathrm{NC}$ group (all $\mathrm{P}<0.05$ ). Administration of klotho, however, 


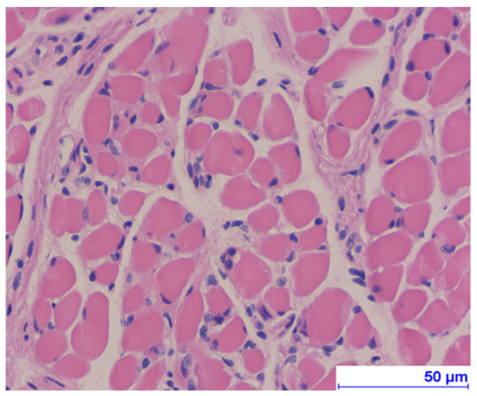

$\mathrm{NC}$

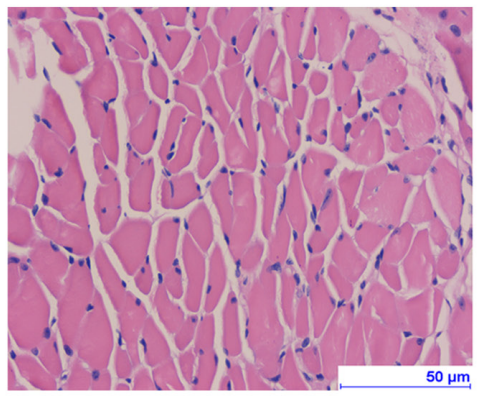

$\mathrm{CIH}$

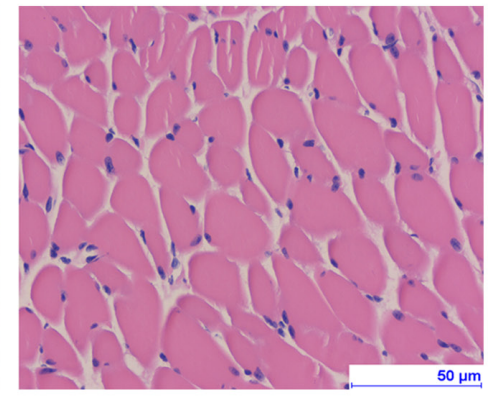

$\mathrm{ClH}+\mathrm{klotho}$

Figure 3. Histological changes in genioglossus muscle samples. Histological changes were detected by hematoxylin and eosin staining in genioglossus muscle samples. Scale bar, $50 \mu \mathrm{m}$. NC, normoxia control; $\mathrm{CIH}$, chronic intermittent hypoxia.

A

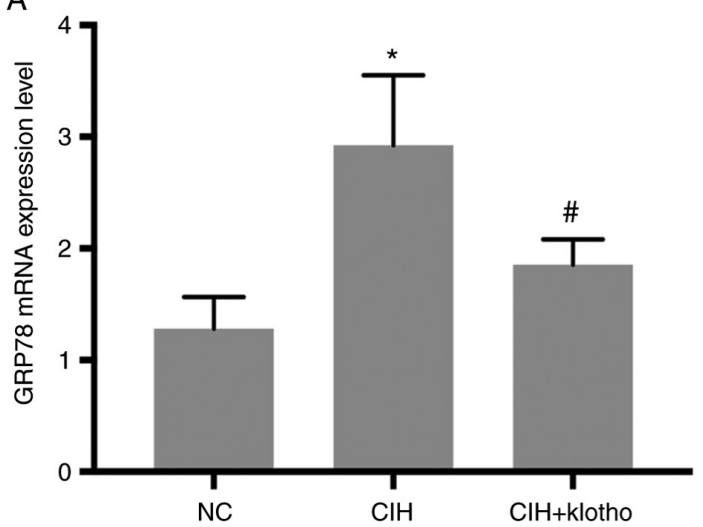

B

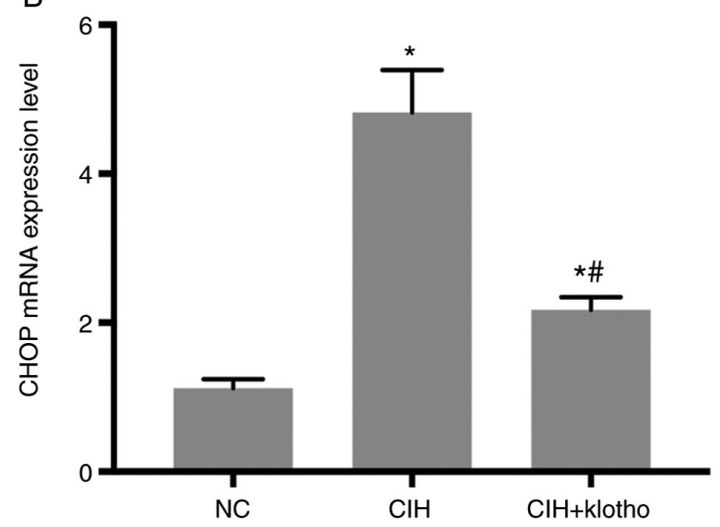

Figure 4. Gene expression levels of endoplasmic reticulum stress-related molecules in genioglossus muscle samples. (A) GRP78 and (B) CHOP mRNA levels were assessed by reverse transcription-quantitative PCR. ${ }^{*} \mathrm{P}<0.05$ vs. CIH group; ${ }^{*} \mathrm{P}<0.05$ vs. NC group. NC, normoxia control; CIH, chronic intermittent hypoxia; GRP78, glucose-regulated protein 78; CHOP, C/EBP homologous protein.

led to a significant reduction in these levels in the model mice (all $\mathrm{P}<0.05$ ). The cleaved caspase-12/caspase-12 ratio remained significantly lower in the $\mathrm{CIH}+$ klotho group compared with the NC group (both $\mathrm{P}<0.05$ ), whereas the levels of GRP78, CHOP and cleaved caspase-3/caspase-3 ratio were similar between the $\mathrm{NC}$ and $\mathrm{CIH}+$ klotho groups (all P>0.05; Fig. 5).

ROS levels in the genioglossus muscle samples. At the end of the 12-week study, compared with the NC group, the $\mathrm{CIH}$ and $\mathrm{CIH}+$ klotho treatment groups were found to have significantly increased ROS levels in the genioglossus muscle samples (all $\mathrm{P}<0.05$ ). Compared with the $\mathrm{CIH}$ group, however, the CIH + klotho group exhibited significantly decreased ROS levels, although they remained higher compared with those of the NC group $(\mathrm{P}<0.05$; Fig. 6$)$.

\section{Discussion}

The present study demonstrated that exogenous klotho may alleviate apoptosis of genioglossus myocytes in mice by inhibiting ROS-associated ERS. As revealed by the experiments described in the present study, the serum and genioglossus muscle tissue klotho protein levels in the CIH group were significantly lower compared with those of the control group, suggesting that the $\mathrm{CIH}$ model had indeed been successfully established, also corroborating previous findings of reduced klotho protein amounts in OSAS (19). Interestingly, after repeated treatment with klotho, the protein was identified both in the serum and in the genioglossus muscle, although at levels lower than normal physiological levels.

Subsequently, the extent of apoptosis of the genioglossus muscle was assessed. Significantly higher apoptotic rates in the $\mathrm{CIH}$ group were identified compared with the control animals, a phenomenon that was slightly, although not completely, reversed by administration of exogenous klotho protein in vivo. These findings confirmed the antiapoptotic effects of klotho $(24,25)$.

Klotho is an anti-aging gene, which has been identified at reduced levels in patients with OSAS, type 2 diabetes mellitus, and in subjects who are smokers $(20,26,27)$. High klotho expression levels in the plasma are associated with good response in patients with acute ischemia stroke (28). Furthermore, it has been reported that increases in the klotho protein level may have a renoprotective function in patients with diabetes and chronic kidney disease (20). Liu et al (22) reported that klotho reduced lipopolysaccharide-induced acute cardiorenal injury in mice. Therefore, it was hypothesized that supplementation with klotho may also protect against $\mathrm{CIH}$-induced injury.

It has been demonstrated that klotho protein is involved in ERS regulation, reducing the pathophysiological injury caused 


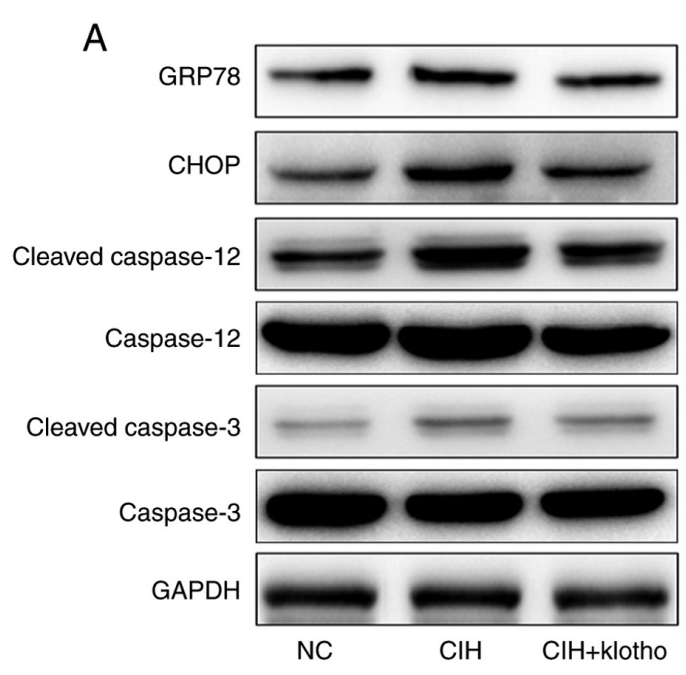

D

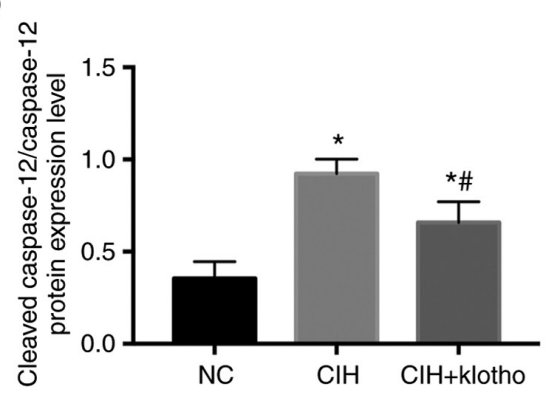

B

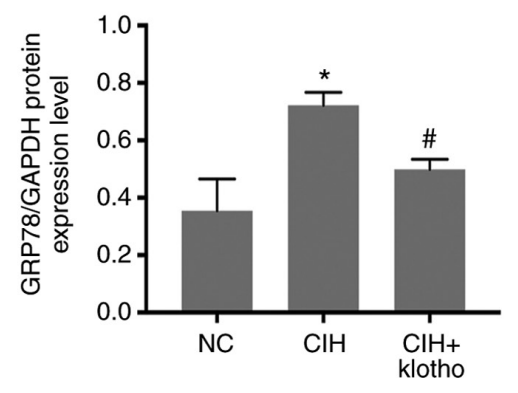

C

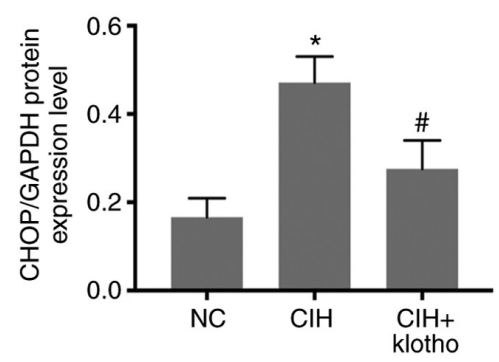

E

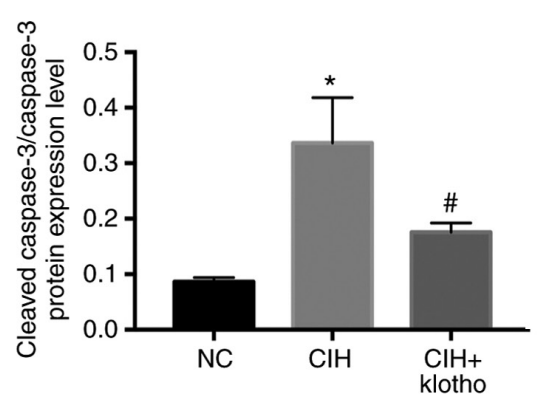

Figure 5. Protein expression levels of endoplasmic reticulum stress-related molecules in genioglossus muscle samples. Protein expression in genioglossus muscle samples was assessed by immunoblotting. (A) GRP78, CHOP, cleaved caspase-12, cleaved caspase-3 and GAPDH immunoreactive bands. Quantitation of (B) GRP78, (C) CHOP, (D) cleaved caspase-12/caspase-12 and (E) cleaved caspase-3/caspase-3 are shown. GAPDH was used as a loading control. ${ }^{*} \mathrm{P}<0.05$ vs. $\mathrm{CIH}$ group; ${ }^{\sharp} \mathrm{P}<0.05$ vs. $\mathrm{NC}$ group. $\mathrm{NC}$, normoxia control; $\mathrm{CIH}$, chronic intermittent hypoxia; GRP78, glucose-regulated protein 78 ; CHOP, $\mathrm{C} / \mathrm{EBP}$ homologous protein.

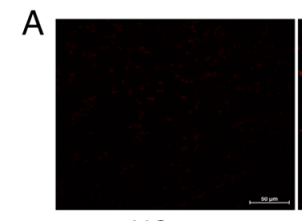

NC

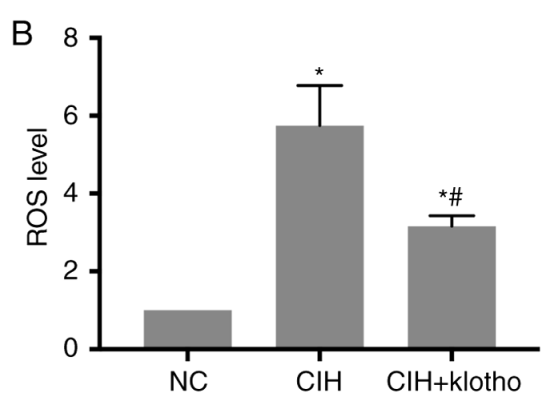

Figure 6. ROS levels in genioglossus muscle samples. ROS levels were quantitated with a DHE assay kit. (A) Micrographs of DHE staining. Scale bar, $50 \mu \mathrm{m}$. (B) Quantitation of A. ${ }^{*} \mathrm{P}<0.05$ vs. CIH group; ${ }^{\#} \mathrm{P}<0.05$ vs. NC group. NC, normoxia control; $\mathrm{CIH}$, chronic intermittent hypoxia; DHE, dihydroethidium; ROS, reactive oxygen species.

by an increased UPR and abnormal ER activation (29). A recent study also demonstrated that activated IRE-1 is coupled with c-Jun N-terminal kinase activation through interaction with TRAF-2 and apoptotic signal-regulated kinase-1 (30). Therefore, whether ERS is involved in CIH-induced apoptosis was the major objective of the present study.

In the present study, establishment of CIH led to an increase in the mRNA expression levels of ERS-associated genes, including GRP78 and CHOP, as well as the protein levels of GRP78, CHOP, cleaved caspase-12 and cleaved caspase-3 in genioglossus muscle samples from mice. These findings were in line with previous reports showing that ERS mediates cell apoptosis to cause cognitive dysfunction in OSAS $(31,32)$. As demonstrated above, administration of exogenous klotho protein significantly reduced the levels of ERS-associated genes and proteins, indicating that klotho protein is involved in regulating CIH-induced, ERS-associated apoptosis.

As oxidative stress is also an important factor in the pathophysiology of airway diseases (7), the present study sought to determine whether the latter was affected by klotho protein treatment in the current model. As shown above, the ROS levels were increased after CIH modeling, but decreased by klotho protein administration. These results indicated that klotho protein alleviated oxidative stress in the CIH model. Taken together, the present findings have demonstrated that exogenous klotho protein inhibits 
the apoptosis levels of genioglossus myocytes in mice with $\mathrm{CIH}$ via suppression of the ROS-associated ERS pathways, and this should be further assessed in order to improve the clinical treatment of OSAS.

The limitations of the present study should, however, be mentioned. First, these experiments were conducted in a mouse $\mathrm{CIH}$ model, and whether similar findings would be obtained in a human study remains unclear. In addition, the animals were treated for a relatively long time, and the observed effects were still not complete. Furthermore, the mice were not assessed for disease characteristics or evaluated after discontinuation of the treatment. Finally, tauroursodeoxycholate (TUDCA) is an ER stress inhibitor, where it remains unknown whether TUDCA decreased klotho-induced oxidative stress and apoptosis. Therefore, the lack of a TUDCA group is another limitation of this study. Therefore, further studies are required to confirm these findings before performing clinical trials that may pave the way for the use of klotho protein in treatment of OSAS in the future.

In conclusion, the present study has demonstrated that exogenous klotho protein may reduce $\mathrm{CIH}$-induced genioglossus muscle injury in mice, at least in part by regulating ERS-associated apoptotic pathways. Further studies, however, are required to corroborate these findings and to help determine whether klotho protein administration may be a feasible option for the treatment of OSAS.

\section{Acknowledgements}

Not applicable.

\section{Funding}

The present study supported by the Nanjing Science and Technology Development Plan Project (20150409-2).

\section{Availability of data and materials}

All data generated or analyzed during this study are included in this published article.

\section{Authors' contributions}

QZ conceived and supervised the study; ZX and QZ designed the experiments; WD and LG performed the experiments; QZ provided new tools and reagents; $\mathrm{ZX}$ developed new software and performed simulation studies; ZX analyzed the data; ZX wrote the manuscript; $\mathrm{ZX}$ made manuscript revisions. All authors have seen and can confirm the authenticity of the raw data. All authors have reviewed the results and approved the final version of the manuscript.

\section{Ethics approval and consent to participate}

This study was approved by the Experimental Animal Ethics Committee of Southeast University (Nanjing, China).

\section{Patient consent for publication}

Not applicable.

\section{Competing interests}

The authors declare that they have no competing interests.

\section{References}

1. Mirrakhimov AE, Sooronbaev T and Mirrakhimov EM: Prevalence of obstructive sleep apnea in Asian adults: A systematic review of the literature. BMC Pulm Med 13: 10, 2013.

2. De Backer W: Obstructive sleep apnea/hypopnea syndrome. Panminerva Med 55: 191-195, 2013.

3. Zhang XF, Huang HP, Ding WX, Ding N, Lu G, Liu JN and Zhang XL: Adiponectin protects the genioglossus of rats against chronic intermittent hypoxia-induced injury via inhibition of endoplasmic reticulum stress. Chin Med J (Engl) 126: 3270-3275, 2013.

4. Wang W, Ding W, Huang H, Zhu Y, Ding N, Feng G and Zhang X: The role of mitophagy in the mechanism of genioglossal dysfunction caused by chronic intermittent hypoxia and the protective effect of adiponectin. Sleep Breath 2020 (Epub ahead of print).

5. Ding WH, Li W, Chen XY and Shi JJ: The study of genistein attenuating genioglossus muscle fatigue under chronic intermittent hypoxia. Zhonghua Kou Qiang Yi Xue Za Zhi 51: 46-50, 2016 (In Chinese).

6. Huang H, Zhang X, Ding N, Li Q, Min Y and Zhang X: Effects of chronic intermittent hypoxia on genioglossus in rats. Sleep Breath 16: 505-510, 2012.

7. Prakash YS, Pabelick CM and Sieck GC: Mitochondrial dysfunction in airway disease. Chest 152: 618-626, 2017.

8. Tabas I and Ron D: Integrating the mechanisms of apoptosis induced by endoplasmic reticulum stress. Nat Cell Biol 13: 184-190,2011.

9. Hetz C: The unfolded protein response: Controlling cell fate decisions under ER stress and beyond. Nat Rev Mol Cell Biol 13: 89-102, 2012

10. Bouvier N, Fougeray S, Beaune P, Thervet E and Pallet N: The unfolded protein response regulates an angiogenic response by the kidney epithelium during ischemic stress. J Biol Chem 287: 14557-14568, 2012.

11. Gardner BM, Pincus D, Gotthardt K, Gallagher CM and Walter P: Endoplasmic reticulum stress sensing in the unfolded protein response. Cold Spring Harb Perspect Biol 5: a013169, 2013.

12. Marciniak SJ, Yun CY, Oyadomari S, Novoa I, Zhang Y, Jungreis R, Nagata K, Harding HP and Ron D: CHOP induces death by promoting protein synthesis and oxidation in the stressed endoplasmic reticulum. Genes Dev 18: 3066-3077, 2004.

13. Zong WX, Li C, Hatzivassiliou G, Lindsten T, Yu QC, Yuan J and Thompson CB: Bax and Bak can localize to the endoplasmic reticulum to initiate apoptosis. J Cell Biol 162: 59-69, 2003.

14. Shore GC, Papa FR and Oakes SA: Signaling cell death from the endoplasmic reticulum stress response. Curr Opin Cell Biol 23: 143-149, 2011.

15. Shi Z, Xu L and Zhou R: Tauroursodeoxycholic acid suppresses endoplasmic reticulum stress in pulmonary tissues of intermittent hypoxia mice. Zhong Nan Da Xue Xue Bao Yi Xue Ban 40: 1165-1172, 2015 (In Chinese)

16. Cui W, Leng B, Liu W and Wang G: Suppression of apoptosis in human umbilical vein endothelial cells (HUVECs) by klotho protein is associated with reduced endoplasmic reticulum oxidative stress and activation of the PI3K/AKT pathway. Med Sci Monit 24: 8489-8499, 2018.

17. Maekawa Y, Ohishi M, Ikushima M, Yamamoto K, Yasuda O, Oguro R, Yamamoto-Hanasaki H, Tatara Y, Takeya Y and Rakugi H: Klotho protein diminishes endothelial apoptosis and senescence via a mitogen-activated kinase pathway. Geriatr Gerontol Int 11: 510-516, 2011.

18. Yamamoto M, Clark JD, Pastor JV, Gurnani P, Nandi A, Kurosu H, Miyoshi M, Ogawa Y, Castrillon DH, Rosenblatt KP and Kuro-o M: Regulation of oxidative stress by the anti-aging hormone klotho. J Biol Chem 280: 38029-38034, 2005.

19. Pákó J, Kunos L, Mészáros M, Tárnoki DL, Tárnoki ÁD, Horváth I and Bikov A: Decreased levels of anti-aging klotho in obstructive sleep apnea. Rejuvenation Res 23: 256-261, 2020.

20. Navarro-González JF, Sánchez-Niño MD, Donate-Correa J, Martín-Núñez E, Ferri C, Pérez-Delgado N, Górriz JL, Martínez-Castelao A, Ortiz A and Mora-Fernández C: Effects of pentoxifylline on soluble klotho concentrations and renal tubular cell expression in diabetic kidney disease. Diabetes Care 41: 1817-1820, 2018. 
21. Song S, Gao P, Xiao H, Xu Y and Si LY: Klotho suppresses cardiomyocyte apoptosis in mice with stress-induced cardiac injury via downregulation of endoplasmic reticulum stress. PLoS One 8: e82968, 2013.

22. Liu X, Niu Y, Zhang X, Zhang Y, Yu Y, Huang J, Li J and Yu C: Recombinant $\alpha$-klotho protein alleviated acute cardiorenal injury in a mouse model of lipopolysaccharide-induced septic cardiorenal syndrome type 5. Anal Cell Pathol (Amst) 2019: 5853426,2019

23. Livak KJ and Schmittgen TD: Analysis of relative gene expression data using real-time quantitative PCR and the 2(-Delta Delta C(T)) method. Methods 25: 402-408, 2001.

24. Cui W, Leng B and Wang G: Klotho protein inhibits $\mathrm{H}_{2} \mathrm{O}_{2}$-induced oxidative injury in endothelial cells via regulation of PI3K/AKT/Nrf2/HO-1 pathways. Can J Physiol Pharmacol 97: 370-376, 2019

25. Mencke R and Hillebrands JL; NIGRAM consortium: The role of the anti-ageing protein Klotho in vascular physiology and pathophysiology. Ageing Res Rev 35: 124-146, 2017.

26. Zhang L and Liu T: Clinical implication of alterations in serum Klotho levels in patients with type 2 diabetes mellitus and its associated complications. J Diabetes Complications 32: 922-930, 2018.

27. Patel MS, Donaldson AV, Lewis A, Natanek SA, Lee JY, Andersson YM, Haji G, Jackson SG, Bolognese BJ, Foley JP, et al: Klotho and smoking-An interplay influencing the skeletal muscle function deficits that occur in COPD. Respir Med 113: 50-56, 2016.
28. Lee JB, Woo HG, Chang Y, Jin YM, Jo I, Kim J and Song TJ: Plasma Klotho concentrations predict functional outcome at three months after acute ischemic stroke patients. Ann Med 51: 262-269, 2019.

29. Banerjee S, Zhao Y, Sarkar PS, Rosenblatt KP, Tilton RG and Choudhary S: Klotho ameliorates chemically induced endoplasmic reticulum (ER) stress signaling. Cell Physiol Biochem 31: 659-672, 2013

30. Song J, Park KA, Lee WT and Lee JE: Apoptosis signal regulating kinase 1 (ASK1): Potential as a therapeutic target for Alzheimer's disease. Int J Mol Sci 15: 2119-2129, 2014.

31. Cai XH, Li XC, Jin SW, Liang DS, Wen ZW, Cao HC, Mei HF, Wu Y, Lin ZD and Wang LX: Endoplasmic reticulum stress plays critical role in brain damage after chronic intermittent hypoxia in growing rats. Exp Neurol 257: 148-156, 2014.

32. Zhou L, Chen P, Peng Y and Ouyang R: Role of oxidative stress in the neurocognitive dysfunction of obstructive sleep apnea syndrome. Oxid Med Cell Longev 2016: 9626831, 2016.

This work is licensed under a Creative Commons

Attribution-NonCommercial-NoDerivatives 4.0 International (CC BY-NC-ND 4.0) License. 